\title{
The Divergence of the Viscosity of a Fluid of Hard Spheres as an Indicator for the Fluid-Solid Phase Transition
}

\author{
Hyearn-Maw Koo and Siegfried Hess \\ Institut für Theoretische Physik, Technische Universität, Berlin, Germany \\ Z. Naturforsch. 42 a, 231-235 (1987); received November 20, 1986 \\ Solution of the Kirkwood-Smoluchowski equation for a hard sphere fluid yields an expression \\ for the viscosity which shows a dramatic pretransitional increase and a divergence at a number \\ density close to that one observed in computer simulations and in colloidal dispersions. The value \\ for the transition density stems from a boundary condition at the surface of the hard sphere in \\ the configurational relative pair-space and makes use of the density dependence of the pair- \\ correlation function and of its derivative at the point of contact.
}

Both computer calculations [1] and experiments with dispersions of colloidal particles [2] show that a fluid of hard spheres (under pressure) undergoes a phase transition liquid-solid at a number density (corresponding to a volume fraction of about 0.5 ) considerably lower than the close packing density (corresponding to the value 0.74 ). It is the purpose of this paper to point out that a theoretical transition density close to the observed value can be inferred from the divergence of the viscosity as based on the Kirkwood-Smoluchowski (KS) equation [3]. Use is made of the density dependence of the Wertheim-Thiele equilibrium pair-correlation function [4] which - as such - does not give an indication of the phase transition fluid-solid. The pretransitional increase of the viscosity is reminiscent of the phenomenological expression due to Hildebrand [5].

\section{Kinetic Equation for the Pair-Correlation Function}

The point of departure is the KS-equation for the pair-correlation function $g$ which depends on the time $t$ and the vector $\vec{r}$ pointing from a reference particle to any other particle in the fluid. A dense fluid composed of spherical particles is considered first. The restriction to hard spheres is made later. In the presence of a linear flow velocity $\vec{v}=\vec{r} \cdot(\vec{\nabla} \vec{v})$

Reprint requests to Prof. Dr. S. Hess, Institut für Theoretische Physik, Technische Universität Berlin, PN 7-1, D-1000 Berlin 12. where $\vec{\nabla} \vec{v}$ is the constant velocity gradient tensor, the KS-equation can be written as [3]

$$
\frac{\partial g}{\partial t}+\vec{v} \cdot \frac{\partial}{\partial \vec{r}} g+\frac{\partial}{\partial \vec{r}} \cdot \vec{j}=0 .
$$

The excess probability current density $\vec{j}$ is given by

$$
\vec{j}=-\tau_{0}^{-1}\left[\frac{\partial}{\partial \vec{r}} g+\left(k_{\mathrm{B}} T\right)^{-1} g \frac{\partial}{\partial \vec{r}} w\right] .
$$

In (2), $T$ is the temperature, $k_{\mathrm{B}}$ is Boltzmann's constant; the effective potential $w$ is related to the equilibrium pair-correlation function $g_{\text {eq }}(r)$ by

$$
w(r)=-k_{\mathrm{B}} T \ln g_{\mathrm{eq}},
$$

and $\tau_{0}$ is a relaxation time linked with a (self) diffusion coefficient $D$ and a friction coefficient $\zeta$ by ( $m$ is the mass of a particle)

$$
\tau_{0}^{-1}=2 D r_{0}^{-2}=2 k_{\mathrm{B}} T(m \zeta)^{-1} r_{0}^{-2} .
$$

Here $r_{0}$ is conveniently chosen reference length and it is understood that $\vec{r}$ occuring in (1) and (2) is expressed in units of $r_{0}$.

The main point to be presented here is the dramatic pretransitional increase of the viscosity which follows from the ansatz for the intermolecular potential consisting of a hard core and an attractive soft force, from the boundary conditions for $\vec{j}$ and the density dependence of $g_{\text {eq }}$ even for the case where the density dependence of $\tau_{0}$ is disregarded.

In previous studies on the flow-induced distortion of the structure of a fluid [6] and on the nonNewtonian viscosity [7] the attention was focussed 
on the effects caused by the flow term in (1), whereas the "damping term" of the KS-equation was simplified. Extensions and modifications $[8,9]$ of the KS-equation are disregarded here.

Next, $g$ is written as

$$
g=g_{\text {eq }}+g_{\text {eq }}^{1 / 2} \Psi,
$$

where $\Psi$ is a measure for the deviation of $g$ from the equilibrium pair-correlation function $g_{\text {eq }}$. The quantity $\Psi$ is needed for the evaluation of the viscosity. Insertion of (5) into (1) with (2) and decomposition of the velocity gradient tensor $\vec{\nabla} \vec{v}$ into its antisymmetric part $\vec{\omega}=1 / 2 \vec{\nabla} \times \vec{v}$ (vorticity) and its symmetric traceless part $\vec{\gamma}$ (deformation rate) leads to

$$
\begin{aligned}
\tau_{0}\left(\frac{\partial}{\partial t}+\vec{\omega} \cdot \vec{f}\right) \Psi-(\Delta+V) \Psi+\tau_{0} g_{\mathrm{eq}}^{-1 / 2} \overleftrightarrow{\gamma}: \vec{f}\left(g_{\mathrm{eq}}^{1 / 2} \Psi\right) \\
=-2 \tau_{0} \overleftrightarrow{\gamma}: \vec{f} g_{\mathrm{eq}}^{1 / 2}
\end{aligned}
$$

Here $\vec{f}$ stand for $\vec{r} \times \partial / \partial \vec{r}$ and $\vec{f}$ is the symmetric traceless part of $\vec{r} \partial / \partial \vec{r}$. Use has been made of the fact that $g_{\text {eq }}$ depends only on the magnitude $r$ of $\vec{r}$. The quantity $V=V(r)$ stands for

$$
\begin{aligned}
V(r) & =\left(2 k_{\mathrm{B}} T\right)^{-1} \Delta w-\left(2 k_{\mathrm{B}} T\right)^{-2}\left(w^{\prime}\right)^{2} \\
& =g_{\mathrm{eq}}^{-1 / 2} \Delta\left(g_{\mathrm{eq}}^{1 / 2}\right),
\end{aligned}
$$

$\Delta=\frac{\partial}{\partial \vec{r}} \cdot \frac{\partial}{\partial \vec{r}}$, and the prime denotes the differentiation with respect to $r$.

\section{Approximations and Special Geometry}

Next, the solution of the inhomogeneous equation (6) is studied for a stationary situation $(\partial \Psi / \partial t=0)$ and in lowest order in the deformation rate tensor $\vec{\gamma}$. The special case of plane Couette flow with the velocity in $x$-direction and its gradient in $y$-direction and the (constant) shear rate $\gamma=\partial v_{x} / \partial y$ is considered next. The usual polar coordinates $r, \vartheta, \varphi$ are used. An expansion with respect to the deformation rate,

and

$$
\Psi=\sum_{n=0}^{\infty}\left(\frac{1}{2} \gamma \tau_{0}\right)^{n} \Psi_{n}
$$

$$
\Psi_{1}=\sum_{m=-\infty}^{\infty} \Psi_{1}^{(2 m)}(r, \vartheta) e^{i 2 m \varphi},
$$

leads to a differential equation for the first order 2-dimensional perturbation function from (6), viz.

$$
\begin{aligned}
& \left(\mathscr{f}_{r}+r^{-2} \mathcal{f}_{\vartheta}+i m \tau_{0} \gamma\right) \Psi_{1}^{(2 m)} \\
& \quad=\left(\delta_{m, 1}-\delta_{m,-1}\right)\left(-i \sin ^{2} \vartheta\right) r\left(g_{\mathrm{eq}}^{1 / 2}\right)^{\prime},
\end{aligned}
$$

where

$$
\begin{aligned}
& \mathcal{L}_{r}=r^{-2} \frac{\partial}{\partial r}\left(r^{2} \frac{\partial}{\partial r}\right)+V(r) \\
& \mathcal{L}_{\vartheta}=(\sin \vartheta)^{-1} \frac{\partial}{\partial \vartheta}(\sin \vartheta) \frac{\partial}{\partial \vartheta}-4 m^{2}\left(\sin ^{2} \vartheta\right)^{-1}
\end{aligned}
$$

Note that the terms involving the shear rate $\gamma$ on the r.h.s. of the first expression of (8) and on the l.h.s. of (9) stem from the deformation and the vorticity, respectively; the latter can be disregarded for a slow flow. It can be shown that the homogeneous solution of the differential equation (9) with the proper boundary conditions vanishes, thus $\Psi_{1}^{(2 m)}=0$ for $m \neq \pm 1$. With the factor $\gamma \tau_{0}$ in (8) (which stems from the deformation rate tensor), the quantity $\Psi_{1}^{(2 m)}$ with $m= \pm 1$ is independent of $\gamma$ in this limit. In the next higher order in the deformation rate, terms with $m=0$ and $m= \pm 2$ are generated from (6) but they are disregarded here.

An explicit solution of (9) can be given when Green's function pertaining to the differential operator and to the boundary conditions, as well as the equilibrium pair-correlation function $g_{\text {eq }}$ are known. The viscosity can then be obtained as an integral over $\Psi_{1}^{( \pm 2)}$.

\section{Viscosity of a Dense Fluid of Hard Spheres}

The potential contribution to the nonequilibrium pressure tensor $\vec{p}$ of a fluid composed of particles interacting with the potential $\phi=\phi(r)$ is

$$
\vec{p}=-\frac{1}{2} n_{0}^{2} r_{0}^{-3} \int \vec{r} \vec{r} r^{-1} \phi^{\prime} g_{\text {eq }}^{1 / 2} \Psi(\vec{r}) \mathrm{d}^{3} r,
$$

where it is understood that $\vec{r}$ is in units of the reference length $r_{0}$ and the number density $n_{0}\left(=n r_{0}^{2}\right)$ in units of $r_{0}^{-3}$. For hard spheres with the diameter $r_{0}$ (the distance of closest approach corresponds to $r=1$ ), one has

$$
\phi^{\prime} g_{\mathrm{eq}}^{1 / 2}(r)=-2 k_{\mathrm{B}} T g_{\mathrm{eq}}^{1 / 2}\left(1^{+}\right) \delta(r-1)
$$

since

$$
\left\{\exp \left[-\phi\left(2 k_{\mathrm{B}} T\right)^{-1}\right]\right\}^{\prime}=\delta(r-1),
$$


and (12) leads to

$$
\vec{p}=n_{0}^{2} r_{0}^{-3} k_{\mathrm{B}} T \int \hat{r} \hat{r} g_{\mathrm{eq}}^{1 / 2}\left(1^{+}\right) \Psi(\hat{r}) d^{2} \hat{r} .
$$

Here $\hat{r}$ stands for the unit vector parallel to $\hat{r}$. The viscosity $\eta$ is inferred from $P_{x y}=-\eta \gamma$; for its calculation the quantity $\Psi$, cf. (8), has to be obtained from the solution of (9). With $V(r)$ in (10) treated as a perturbation, Green's function [10] to the differential equation (9) can be calculated for hard spheres subject to the boundary conditions

$\vec{j}(r) \rightarrow 0$ for $r \rightarrow \infty$ and $\hat{r} \cdot \vec{j}=0$ for $r=1$;

$\vec{j}$ is the excess probability current, cf. (2). In particular, for $V=0$ and $\gamma \rightarrow 0$ one finds [10] for the "potential contribution" to the viscosity

$$
\eta=n_{0} k_{\mathrm{B}} T \tau_{0} \frac{8}{15} \times\left(g_{\mathrm{eq}}\left(1^{+}\right)\right)^{1 / 2} \Lambda^{-1},
$$

with the volume fraction $x=n r_{0}^{3} \pi / 6=n_{0} \pi / 6$ and

$$
\begin{aligned}
\Lambda & =1-\left(6 k_{\mathrm{B}} T\right)^{-1} w^{\prime}\left(1^{+}\right) \\
& =1+(1 / 6) g_{\mathrm{eq}}^{\prime}\left(1^{+}\right) / g_{\mathrm{eq}}\left(1^{+}\right) ;
\end{aligned}
$$

for $w$ see (3). The quantity $\Lambda$ as given by (15) stems from the boundary condition at the point of contact $\left(r=1^{+}\right)$. Equation (14) is an expression for the Newtonian viscosity at small shear rates. Terms involving powers of $V(r)$ from (10) will give additional contributions to the viscosity but are not expected to remove the singularity at $\Lambda=0$ which is looked upon as an indicator for the fluid-solid phase transition.

\section{Volume Fraction at the Fluid-Solid Phase Transition}

Due to (3) and (15), the condition $\Lambda=0$ corresponds to

$$
6 g_{\text {eq }}\left(1^{+}\right)+g_{\text {eq }}^{\prime}\left(1^{+}\right)=0 .
$$

Insertion of the exact solution [4] of the PercusYevick equation,

and

$$
g_{\text {eq }}\left(1^{+}\right)=(1+1 / 2 x)(1-x)^{-2},
$$

$$
g_{\text {eq }}^{\prime}\left(1^{+}\right)=-9 / 2 x(1+x)(1-x)^{-3}
$$

into (16) leads to the equation $5 x^{2}+5 x-4=0$, which, in turn, yields the value

$$
x_{t}=(1.05)^{1 / 2}-0.5 \approx 0.524695
$$

for the volume fraction $x$ at the fluid-solid phase transition as inferred from the divergence of the viscosity. The theoretical value (17) is remarkably close to the computer results [1] which are $x \approx 0.494$ and $x \approx 0.545$ for the volume fractions of the fluid and the coexisting fcc solid of hard spheres; the densest packing corresponds to 0.7405 . Experiments [2] on dispersions of (almost) hard spheres also yield $x \approx 0.5$ for the transition value of the volume fraction.

It is to be noticed that the condition (16) is independent of the approximations which were introduced to calculate the viscosity coefficient (14). It is associated with the position of the singularity of the viscosity coefficient, which stems from the boundary condition at $r=1^{+}$. Moreover, the condition (16) is also valid for molecules whose intermolecular potential is composed of a hard core and a week attractive force.

\section{Density Dependence of the Viscosity}

In Fig. 1, the "KS-viscosity" given by (14) is plotted as function of the volume fraction $x$. For comparison, the kinetic (direct momentum transfer: DMT) and collisional transfer (CT) contributions of the Enskog viscosity $[14,15]$ are also shown. All viscosity coefficients are in units of $m c_{0} d^{-2}$, where $d$ is the diameter of a hard sphere and $c_{0}=$ $\left(k_{\mathrm{B}} T\right)^{1 / 2} m^{-1 / 2}$. The (reference) relaxation time $\tau_{0}$ occurring in (14) was specified as

$$
\tau_{0}=\frac{1}{3} c_{0}^{-2} \omega_{\mathrm{B}} d^{2}
$$

with the Boltzmann collision frequency

$$
\omega_{\mathrm{B}}=4 \pi^{1 / 2} n c_{0} d^{2} ;
$$

$n$ is the number density. Relation (18) follows from (4) with $r_{0}=d$ and with the Boltzmann expression $D=\frac{3}{2} c_{0}^{2} \omega_{\mathrm{B}}^{-1}$. The quantity $\chi[14,15]$, which modifies the collision frequency in the Enskog-Boltzmann theory was identified with $g_{\text {eq }}\left(1^{+}\right)$. At intermediate densities $(x \leqq 0.3)$, our "collisional transfer" contribution based on the KS equation does not differ much from the Enskog result. For higher densities $(x \geqq 0.4)$, however, (14) leads to considerably larger values and to a dramatic pretransitional increase, which does not occur for the Enskog viscosity. 


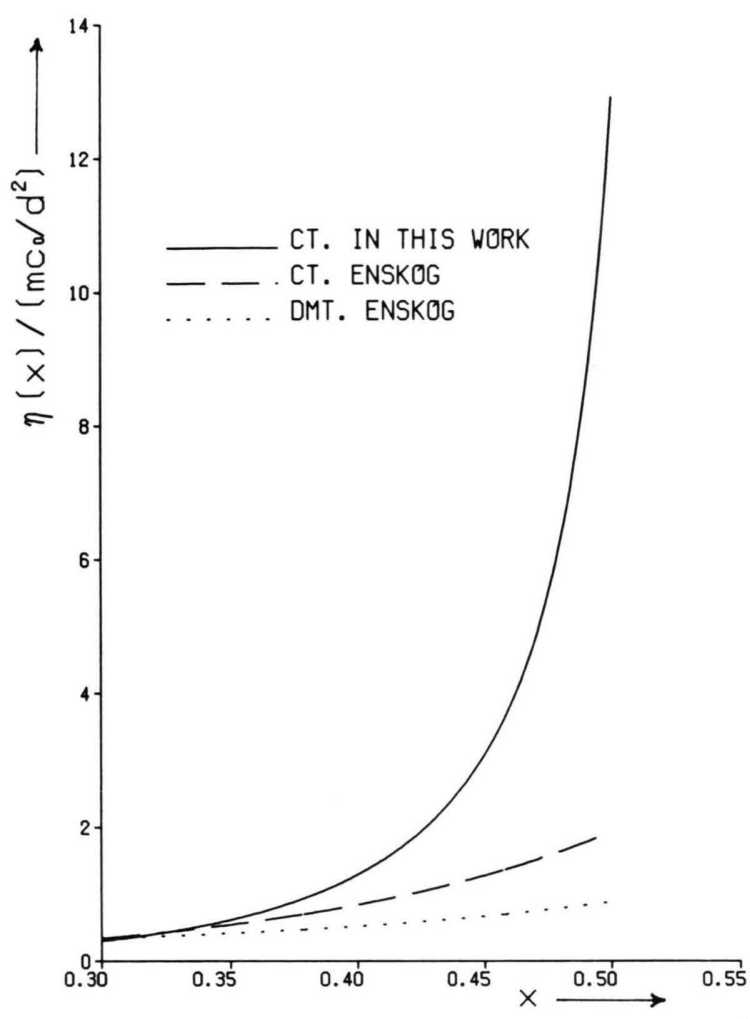

Fig. 1. Contribution to the viscosity $\eta$ in units of $m c_{0} d^{-2}$ as functions of the volume fraction $x=\frac{\pi}{6} n d^{3}$, from $x=0.3$ to $x=0.5$. The full curve is obtained from (14) with (18). The dashed and dotted curves are the collisional and direct momentum transfer contributions due to Enskog.

In Fig. 2, the reciprocal of the sum of the kinetic contribution (DMT) and our viscosity (14) is plotted versus $x^{-1}$, which is proportional to the specific volume (Hildebrand plot). The corresponding Enskog expression - reciprocal of the sum of the kinetic (DMT) and the collisional transfer (CT) contributions of the viscosity - is also displayed. For comparison, experimental data [16] for Argon are shown (stars) where $d$ was identified with the temperature independent Lennard-Jones diameter $(3.4 \AA)$. Note that no adjustable parameter is involved. The agreement can even be improved by introducing a temperature dependent effective hard sphere diameter $d$, e.g. by $\Phi(d)=k_{\mathrm{B}} T$ where $\Phi(r)$ is the Lennard-Jones potential. The approximations which led to (14) seem to be quite acceptable.

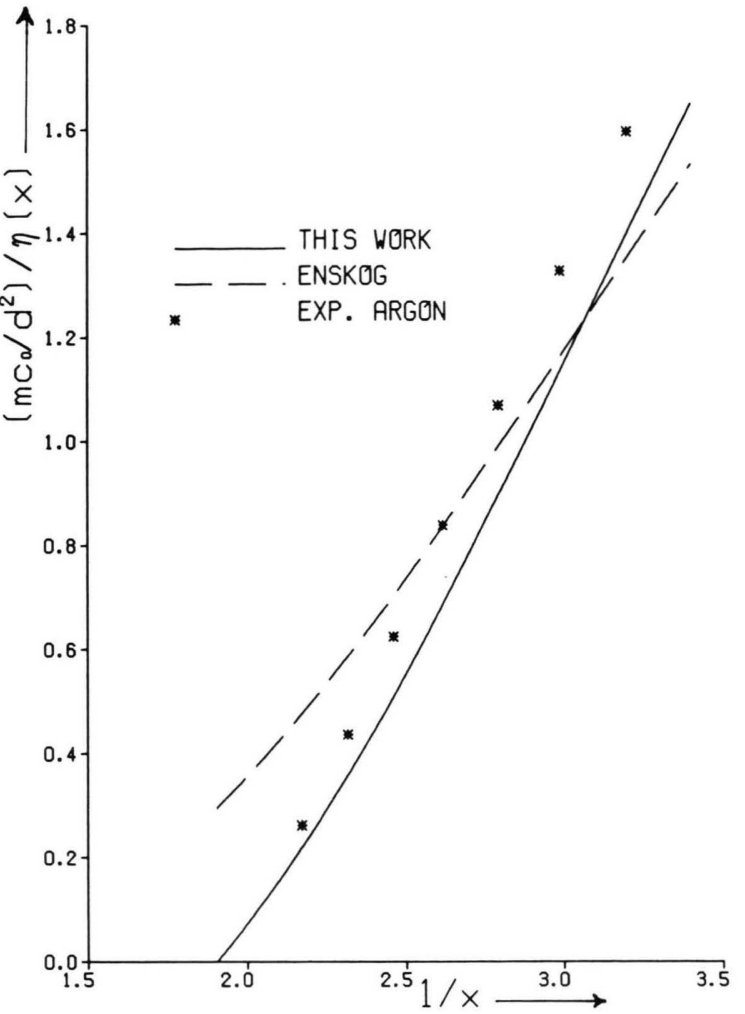

Fig. 2. Hildebrand plot: $\eta^{-1}$ versus $x^{-1}$ for the present theory and the Enskog result. The stars mark experimental points for Argon where $d$ was put equal to the temperature independent Lennard-Jones diameter.

\section{Concluding Remarks}

The expression (14) for the potential contribution to the viscosity of a hard sphere fluid shows a pretransitional increases qualitatively similar to that found in many liquids [2] (under pressure); the divergence of $\eta$ occurs close to the observed transition density.

The present analysis can and indeed been carried out [10] to study nonlinear flow properties: shear rate dependence of the viscosity and normal pressure differences. For small and intermediate shear rates $\gamma$, the non-newtonian viscosity shows shear thinning behavior similar to that found in computer simulations for hard [11] and soft [12,13] spheres. The occurrence of shear-induced spatial structures 
$[11,13]$ is not explained by the present approach. For high shear rates a shear thickening behavior is predicted. An extension of the present theory to fluids of oriented nonspherical particles [17] with applications to nematic liquid crystals is desirable and seems to be feasible.

[1] B. J. Alder and T. Wainwright, J. Chem. Phys. 27, 1209 (1957); W. G. Hoover and F. H. Ree, J. Chem. Phys. 49, 3609 (1968).

[2] C. G. de Kruif, P. W. Rouw, J. W. Jansen, and A. Vrij, J. de Physique 46, C 3-295 (1985); P. N. Pusey and J. G. Rarity, J. de Physique 46, C9-43 (1985); P. N. Pusey and W. van Megen, in: Complex fluids, Proc. eds. J. S. Huang and S. A. Safran, Wiley, New York 1986.

[3] J. G. Kirkwood, J. Chem. Phys. 14, 180 (1946); J. G. Kirkwood, F. B. Buff, and H. M. Green, J. Chem. Phys. 17, 149 (1949).

[4] M. S. Wertheim, Phys. Rev. Lett. 10, 311 (1963); E. Thiele, J. Chem. Phys. 39, 474 (1963); the Wertheim-Thiele pair correlation function becomes negative and this physically unacceptable for densities corresponding to $x \geqq 0.62$.

[5] J. S. Hildebrand and R. H. Lamoreaux, Proc. Nat. Acad. Sci USA 69, 3428 (1972); D. E. Diller, Physica 10 A, 417 (1980).

[6] S. Hess, Phys. Rev. A 22, 2844 (1980); S. Hess and H. J. M. Hanley, Phys. Letters 98 A, 35 (1983); J. F. Schwarzl and S. Hess, Phys. Rev. A 33, 4277 (1986).
Acknowledgement

Financial support by the DAAD is gratefully acknowledged.

[7] S. Hess, Phys. Rev. A 25, 614 (1982); J. C. Rainwater and S. Hess, Physica 118 A, 371 (1983).

[8] T. Ohtsuki, Physica 108 A, 441 (1981).

[9] S. Hess, Physica 118 A, 79 (1983).

[10] H.-M. Koo, Analytic solution for the nonequilibrium pair-correlation function and the non-Newtonian viscosity coefficients of simple liquids, Thesis, University of Erlangen-Nürnberg, 1985, unpublished.

[11] J. J. Erpenbeck, Phys. Rev. Lett. 52, 1333 (1984).

[12] S. Hess and H. J. M. Hanley, Phys. Rev. A 25, 1801 (1982); Int. J. Thermophys. 4, 97 (1983).

[13] S. Hess, Proc. Colloidal crystals, Les Houches, 1984; J. de Physique 46, C 3-191 (1985); Int. J. Thermophys. 6, 657 (1985).

[14] S. Chapman and T. G. Cowling, The mathematical theory of nonuniform gases, Cambridge Univ. Press, 2nd ed. Cambridge 1960.

[15] G. Schmidt, W. E. Köhler, and S. Hess, Z. Naturforsch. 36a, 545 (1981).

[16] B. A. Younglove, J. Phys. and Chem. Ref. Data 111, Suppl. 1, 1982.

[17] D. Baalss and S. Hess, Phys. Rev. Lett. 57, 86 (1986). 